\title{
Uso combinado do tratamento medicamentoso e terapia de Reiki em pacientes com dor no stress pós-traumático
}

\author{
Combined use of drug treatment and Reiki therapy in patients with post-traumatic stress pain \\ Uso combinado de tratamiento farmacológico y terapia Reiki en pacientes con dolor de estrés
} postraumático

Recebido: 02/07/2021 | Revisado: 06/07/2021 | Aceito: 09/07/2021 | Publicado: 17/07/2021

\author{
Livia Dantas Fragoso \\ ORCID: https://orcid.org/0000-0002-7436-7660 \\ Centro Universitário de Patos UNIFIP, Brasil \\ E-mail: liviafragoso@med.fiponline.edu.br \\ Julia Leite Montenegro Pires \\ ORCID: https://orcid.org/0000-0003-4245-7386 \\ Centro Universitário de Patos UNIFIP, Brasil \\ E-mail: juliamontenegropires@gmail.com \\ Marilia Glicia Ferreira \\ ORCID: https://orcid.org/0000-0002-1880-3325 \\ Centro Universitário de Patos UNIFIP, Brasil \\ E-mail: mariliagliciafe@gmail.com \\ Rita De Cássia Pereira Dantas \\ ORCID: https://orcid.org/0000-0002- 1373-6928 \\ Centro Universitário de Patos UNIFIP, Brasil \\ E-mail: ritadantasp@gmail.com \\ Milena Nunes Alves De Sousa \\ ORCID: https://orcid.org/0000-0001-8327-9147 \\ Centro Universitário de Patos UNIFIP, Brasil \\ E-mail: milenanunes@ fiponline.edu.br
}

\begin{abstract}
Resumo
O uso do Reiki vem crescendo nos últimos tempos, sendo uma técnica japonesa que consiste em uma terapia energética. O objetivo é analisar a eficácia da associação do Reiki com a terapia medicamentosa em pacientes com dor e observar como esse tratamento pode amenizar os sintomas. Trata-se de uma revisão sistemática realizada com base em ensaios clínicos randomizados. Realizou-se uma pesquisa naMedical Publishere Biblioteca Virtual em Saúde. Para busca dos artigos utilizaram-se os Descritores em Ciências da Saúde no idioma inglês unidos pelo descritor booleano AND: Reiki e Pain.Dessa forma, os 5artigos foram classificados conforme os critérios estabelecidos no sistema Grading of Recommendations Assessment, Development and Evaluation. A partir dos estudos selecionados observou-se que os grupos oscilaram entre 30 a 90 pacientes, seguido de utilização de opioides, analgésicos e antiinflamatórios para o alívio da dor, associado com o Reiki em tempos de sessões que variaram de 10 a 30 minutos. Além disso, percebeu-se que a associação entre esses e a terapia de Reiki potencializou a eficácia da diminuição da dor, estresse, níveis de ansiedade e tempo de medicação. Alguns estudos, também, se depararam com melhora dos sinais vitais. Concluiu-se que a terapia Reiki associada à terapia medicamentosa, como analgésicos e opioides, tem boa eficácia em pacientes com dor, ficando evidente sua vantagem no uso em indivíduos que precisam de analgesia. Palavras-chave: Ansiedade; Reiki; Dor.
\end{abstract}

\begin{abstract}
The use of Reiki has been growing in recent times, being a Japanese technique consisting of na energy therapy. The aimisto analyze the efficacy of the association of Reiki with drug therapy in patients with pain and observe how this treatment canal leviate the symptoms. Thisis a systematic review conducted on the basis of randomized clinical trials. A research was carried out at the Medical Publisher Virtual Health Library. To search the articles, the Descriptors in Health Sciences in the English language were used by the Booleand escriptor AND: Reiki and Pain. Thus, the 5 articles were classified according to the defined criteria System Classification of Recommendations Evaluation, Development and Evaluation. From the selected studies it was observed that the groupsr anged from 30 to 90 patients, followed by the use of opioids, analgesics and anti-inflammatory drugs for pain relief, associated with Reiki in alternating times ranging from 10 to 30 minutes. Further more, it is induced that the association between these and Reiki therapyh a senhanced the effective ness of decreasing pain, stress, anxiety levels and medication time. In some studies, he also found improvement in vital signs. It was concluded that a Reikit herapy associated with drug therapy,
\end{abstract}


such as analgesics and opioids, has good efficacy in patients with pain, and it's advantage is evident in it's use in patients who need analgesia.

Keywords: Anxiety; Reiki; Pain.

\section{Resumen}

El uso de Reiki ha ido creciendo en los últimos tiempos, siendo una técnica japonesa consistente en la terapia de energía na. El aimisto analizará la eficacia de la asociación del Reiki con la farmacoterapia en pacientes con dolor y observará cómo este canal de tratamiento levita los síntomas. Se trata de una revisión sistemática realizada sobre la base de ensayos clínicos aleatorios. Se llevó a cabo una investigación en la Biblioteca Virtual de Salud de la Editorial Médica. Para la búsqueda de los artículos, los Descriptores en Ciencias de la Salud en el idioma inglés fueron utilizados por el escriptor booleano AND: Reiki and Pain. Así, los 5 artículos fueron clasificados de acuerdo con los criterios definidos Sistema de Clasificación de Recomendaciones Evaluación, Desarrollo y Evaluación. A partir de los estudios seleccionados se observó que el grupo anged de 30 a 90 pacientes, seguido por el uso de opioides analgésicos y antiinflamatorios para el alivio del dolor, asociados a Reiki en tiempos alternos que van de 10 a 30 minutos. Además, se induce que la asociación entre estos y la terapia de Reikisenhanced la eficacia de la disminución del dolor, estrés, niveles de ansiedad y tiempo de medicación. En algunos estudios, también encontró mejoría en signos vitales. Se concluyó que un herapyReikit asociado con la terapia farmacológica, como analgésicos y opioides, tiene buena eficacia en pacientes con dolor, y su ventaja es evidente en su uso en pacientes que necesitan analgesia.

Palabras clave: Ansiedad; Reiki; Dolor.

\section{Introdução}

O uso do Reiki vem crescendo nos últimos tempos, apesar de ser uma terapia antiga ela começou a ser mais realizada nos últimos anos, por essa razão há uma busca de conhecimento a partir de novos estudos, com a finalidade de compreender melhor os benefícios e a importância dessa prática no auxílio da redução da dor. Reiki é uma técnica japonesa que consiste em uma terapia energética, na qual é utilizado as mãos do terapeuta para ajustar o campo de energia do paciente, na tentativa de induzir o corpo a restabelecer o seu equilíbrio (Freitag, Andrade \& Badke, 2015). O Centro Nacional de Saúde Complementar e Integrativa mostra que utilizar técnicas que alteram os campos de energia do corpo pode causar alterações benéficas significativas, como o alívio dos sintomas (Baldwin, Vitale, Brownell, Kryak \& Rand,2017).

Não é uma técnica invasiva, não apresenta efeitos adversos nem contraindicações, além de que estudo mostra sua eficácia no tratamento da ansiedade e dor, como também provoca aumento do sistema imunológico humoral e reduz a pressão arterial, demonstrando que o Reiki está muito relacionado ao relaxamento e aumento da sensação de bem-estar (Weze, Leathard \& Stevens, 2005).Por apresentar respostas no tratamento e prevenção de diversos problemas, seja do âmbito da saúde física, mental ou espiritual, o Reiki vem sendo bastante aceito como forma de tratamento complementar (Freitag, Andrade \& Badke, 2014). É válido destacar, que existem níveis de prática do Reiki, isso é importante para os profissionais que desejam ter conhecimento dessa área. O primeiro grau está relacionado a indivíduos capazes de realizar o Reiki em si mesmo e em outras pessoas, o segundo grau já possibilita que a técnica obtenha êxito mesmo a distância e por fim o terceiro grau, que é alcançado por pessoas que já possuem a capacidade de ensinar essa técnica (Thrane \& Cohen, 2014).

A dor é um fenômeno muito subjetivo que é percebido de diferentes formas pelo paciente, já que cada pessoa significa esse estado de maneira distinta, porém existem alterações comuns que ocorrem nos indivíduos que passam por algum processo pós-traumático, como a elevação da frequência cardíaca, pressão arterial e funcionamento endócrino, sendo nesses casos satisfatória a atuação do Reiki, por ser um procedimento simples e eficiente (Midilli \& Eser, 2015). Um exemplo importante a ser destacado, são os pacientes que se recuperam de um pós-operatório, com alterações da qualidade do sono e transtornos ansiosos. Isso ocorre devido ao estresse pelo qual esses indivíduos foram submetidos, já que a dor é um sintoma frequente e que pode persistir por várias horas, principalmente se houver redução dos analgésicos (Santos, Gomes, Bezerra \& Püschel, 2019). A partir disso, foi notado um aumento do uso da técnica em vários centros hospitalares, nos serviços de emergência e psiquiatria, cirurgia e enfermagem, sendo realizada a capacitação dos profissionais para a realização do Reiki (Spezzia \& Spezzia, 2018). 
Por se tratar de uma sintomatologia muito frequente e que provoca consequências significativas na rotina dos indivíduos acometidos, esse presente estudo tem como objetivo analisar a eficácia da associação do Reiki com a terapia medicamentosa em pacientes com dor e observar como esse tratamento pode amenizar os sintomas desses indivíduos.

\section{Metodologia}

Trata-se de uma revisão sistemática realizada com base em ensaios clínicos randomizados. Tal modalidade de produção científica utiliza de métodos sistemáticos e explícitos com o intuito deentender e definir, com base nos artigos analisados, o que funciona ou não em um determinado contexto, além de avaliar a qualidade e validade das produções científicas estudadas. Constitui um método eficaz para obtenção de meios para a prática baseada em evidências (PBE) (De-LaTorre-Urgarte-Guanilo, Takahasi \& Bertolozzi, 2011; Galvão \& Ricarte, 2019).

A PBE objetiva promover uma melhoria do cuidado, através da identificação e promoção de práticas eficientes e eliminação das ineficientes. Para isso utiliza as etapas de identificação de um problema clínico, formulação de uma questão clínica relevante e específica, busca de evidências científicas e avaliação das evidências disponíveis. Para elaboração desta pergunta utiliza-se a estratégia PICO que representa o acrônimo para Paciente, Intervenção, Comparação e "Outcomes" (desfecho) (Akobeng, 2005). Com base na busca realizada no presente artigo foi formulada a questão PICO: Em pacientes com dor no stress pós-traumático, o do tratamento medicamentoso e terapia de Reiki, em comparação com apenas o uso de fármacos traz benefícios?

Realizou-se uma pesquisa entre os meses de março e maio de 2020 de estudos que abordassem o tema da revisão nas plataformas eletrônicas Medical Publisher (PubMed) e Biblioteca Virtual em Saúde do Ministério da Saúde (BVS), optou-se por tais plataformas por serem de amplo acesso, fácil uso e grande número de publicações de acesso livre e gratuito. Para busca dos artigos utilizaram-se os Descritores em Ciências da Saúde (DeCS) no idioma inglês unidos pelo descritor booleano AND: Reiki e Pain. Aplicou-se essa associação nas bases de literatura eleitas para a pesquisa.

Selecionaram-se os estudos randomizados e ensaios clínicos controlados que abordassem em sua pesquisa a correlação entre o uso associado de Reiki e tratamento medicamentosos para alívio do sintoma de dor em pacientes submetidos a estresse após trauma corporal publicados nos últimos dez anos. Durante a pesquisa foram excluídas pesquisas observacionais, estudos que não responderam ao tema proposto e artigos presentes em ambas as bases de dados utilizadas para pesquisa neste artigo.

A elegibilidade criteriosa dos artigos foi realizada em conjunto com todos os autores desta publicação científica. Em um primeiro momento, foram selecionados todos os artigos de acordo com título e resumo que se respondiam ao questionamento proposto, os artigos selecionados passaram para a segunda etapa, Neste segundo momento, os artigos, inicialmente selecionados, foram lidos em sua totalidade e selecionados conforme consenso comum de todos os autores quanto àcapacidade de responder ao tema proposto.

Tal processo de seleção deve ser explicado na forma de fluxo, conforme as diretrizes da Preferred Reporting Items for Systematic Reviews and Meta-Analyses ou Recomendação PRISMA (Galvão, Pansani \& Harrad, 2015), demonstrada no fluxograma a seguir. 
Fluxograma 1: Etapas seguidas para seleção dos artigos que compõem esse estudo conforme recomendação PRISMA.
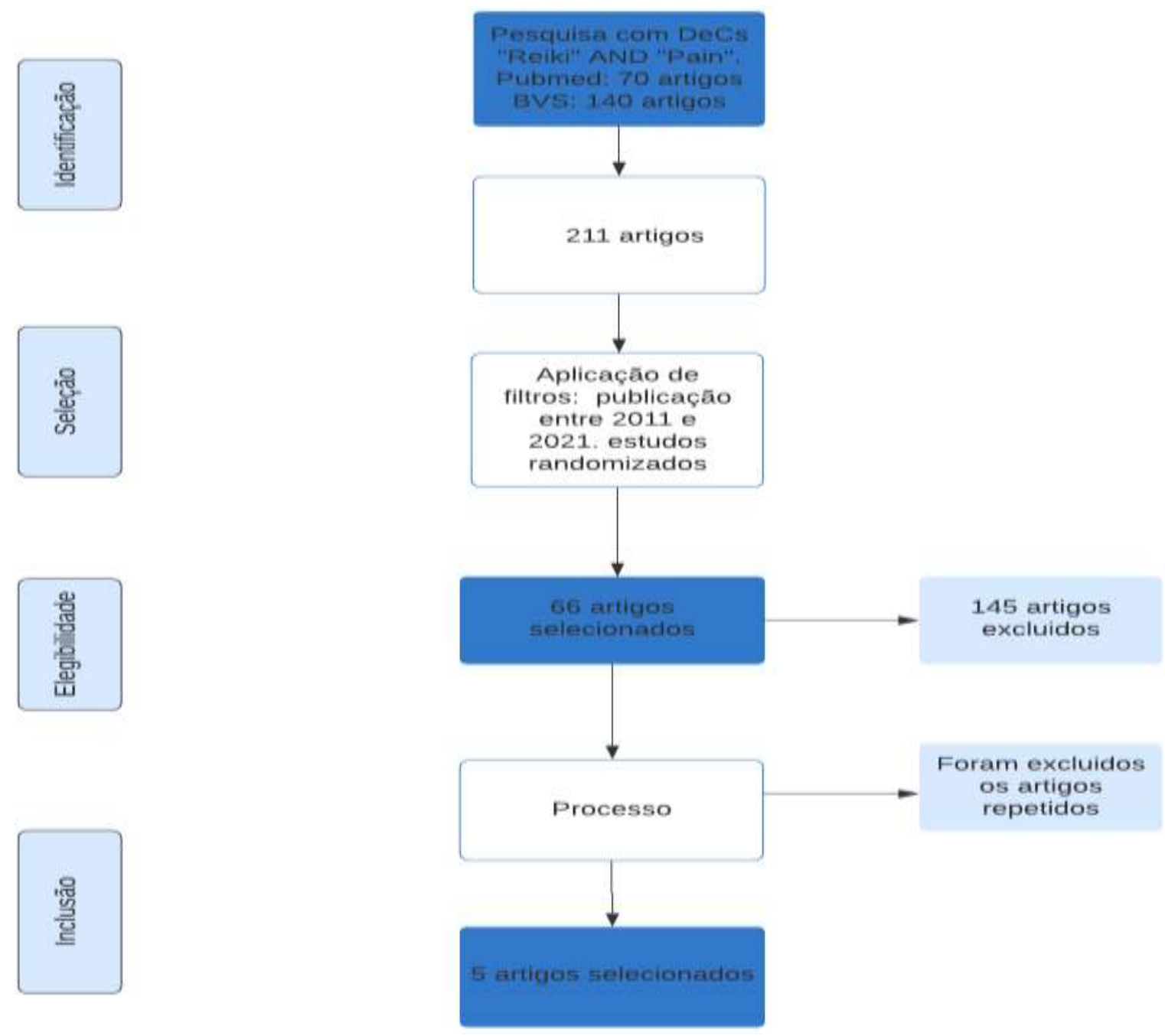

Fonte: Autores (2021).

Em seguida, realizou-se a síntese dos resultados apresentados nesta produção científica. Por fim, realizou-se a síntese dos resultados apresentados nesta produção científica. E, por fim, os artigos foram classificados conforme os critérios estabelecidos sistema Grading of Recommendations Assessment, Development and Evaluation (GRADE) que leva em consideração as limitações metodológicas, as inconsistências, as evidências indiretas e a imprecisão. Após a análise de tais critérios, os estudos são classificados em alto, moderado, baixo ou muito baixo níveis de evidências (Brasil, 2014).

\section{Resultados}

De acordo com o Quadro 1, pode-se observar a distribuição dos estudos quanto ao ano de produção, tipo de estudo, base de dados em que foi encontrado e o país em que foi produzido. Constatou-se que todos os estudos selecionados se tratavam de ensaios clínicos randomizados, e foram provenientes de dois países principais: Estados Unidos da América (EUA) e Turquia. Quanto à base de dados, os cinco estudos puderam ser encontrados na PUBMED e BVS durante as buscas, de acordo com os filtros selecionados. 
Quadro 1: Especificações dos estudos de acordo com o ano da publicação.

\begin{tabular}{|c|c|c|c|c|c|}
\hline Autores (ano) & Título & Tipo de estudo & $\begin{array}{l}\text { Níveis de } \\
\text { evidências }\end{array}$ & $\begin{array}{l}\text { Base de } \\
\text { dados }\end{array}$ & País \\
\hline Baldwin et al.(2017) & $\begin{array}{l}\text { Effects of reiki on pain, anxiety, and } \\
\text { blood pressure in patients undergoing } \\
\text { knee replacement: a pilot study }\end{array}$ & $\begin{array}{l}\text { Ensaio clínico } \\
\text { controlado }\end{array}$ & B & PUBMED & EUA \\
\hline $\begin{array}{l}\text { Bourque, Sullivan \& } \\
\text { Winter (2012) }\end{array}$ & $\begin{array}{l}\text { Reiki as a pain management adjunct in } \\
\text { screening colonoscopy }\end{array}$ & $\begin{array}{l}\text { Ensaio clínico } \\
\text { controlado }\end{array}$ & $\mathrm{B}$ & BVS & EUA \\
\hline Midili et al. (2016) & $\begin{array}{l}\text { Effects of Reiki on Pain and Vital Signs } \\
\text { When Applied to the Incision Area of the } \\
\text { Body After Cesarean SectionSurgery: A } \\
\text { Single-Blinded, Randomized, Double- } \\
\text { Controlled Study. }\end{array}$ & $\begin{array}{l}\text { Ensaio clínico } \\
\text { controlado }\end{array}$ & $\mathrm{B}$ & PUBMED & EUA \\
\hline $\begin{array}{l}\text { Midilli e Eser } \\
\text { (2015) }\end{array}$ & $\begin{array}{l}\text { Effects of Reiki on Post-cesarean } \\
\text { Delivery Pain, Anxiety, and } \\
\text { Hemodynamic Parameters: A } \\
\text { Randomized, Controlled Clinical Trial }\end{array}$ & $\begin{array}{l}\text { Ensaio clínico } \\
\text { randomizado e } \\
\text { controlado }\end{array}$ & B & PUBMED & TURQUIA \\
\hline $\begin{array}{l}\text { Notte, Fazzini \& } \\
\text { Mooney. (2016) }\end{array}$ & $\begin{array}{l}\text { Reiki's Effect on patients with total knee } \\
\text { arthroplasty: A pilot study }\end{array}$ & $\begin{array}{l}\text { Ensaio clínico } \\
\text { controlado }\end{array}$ & B & BVS & TURQUIA \\
\hline
\end{tabular}

Fonte: Autores (2021).

No Quadro 2 estão descritos os dados relacionados ao tipo de tratamento realizado pelos pesquisadores, a quantidade de pacientes submetidos ao uso combinado entre medicamentos e Terapia de Reiki, dose dos medicamentos utilizados, tempo de sessão da terapia e tipo de tratamento responsável pela redução da dor. De acordo com eles, os grupos oscilaram entre 30 a 90 pacientes, seguido de utilização de opioides, analgésicos e antiinflamatórios para o alívio da dor, associado com o Reiki em tempos de sessões que variaram de 10 a 30 minutos. Contatou-se que em todos, o Reiki associado à terapia oral foram responsáveis pela redução da dor. 
Quadro 2: Descrição dos estudos realizados pelos autores quanto ao tratamento.

\begin{tabular}{|c|c|c|c|c|}
\hline Autores/ano & $\begin{array}{c}\text { Grupo } \\
\text { analisado }\end{array}$ & Medicamentos usados & Tempo de sessão & $\begin{array}{l}\text { Medicamento responsável } \\
\text { pela redução da dor }\end{array}$ \\
\hline $\begin{array}{l}\text { Baldwin } \quad \text { et } \\
\text { al. }(2017)\end{array}$ & 46 pacientes & $\begin{array}{l}\text { Morfina }(2 \mathrm{mg}) \\
\text { Oxicodona }(5 \mathrm{mg}) / \text { Acetomifeno } \\
(325 \mathrm{mg})\end{array}$ & 30 min de Reiki & Reiki e Sham Reiki \\
\hline $\begin{array}{l}\text { Bourque et al. } \\
\text { (2012) }\end{array}$ & 30 pacientes & $\begin{array}{l}\text { Doses médias base de Meperidina } \\
50 \mathrm{mg} / 150 \mathrm{mg}\end{array}$ & 10minutos & Analgésicos opioides e Reiki \\
\hline $\begin{array}{l}\text { Midili } \\
(2016)\end{array} \quad$ et. al. & 45 pacientes & Diclofenaco $75 \mathrm{ml} / 3 \mathrm{ml} \mathrm{IM}$ & 15 minutos & $\begin{array}{l}\text { Analgésicos não opioides e } \\
\text { Reiki }\end{array}$ \\
\hline $\begin{array}{l}\text { Midilli } \\
(2015)\end{array}$ e Eser & 90 pacientes & Diclofenaco $75 \mathrm{mg} / 3 \mathrm{~mL}$, IM & 30 minutos & $\begin{array}{l}\text { Analgésicos não opioides e } \\
\text { Reiki }\end{array}$ \\
\hline Notte et al. (2016) & 43 pacientes & $\begin{array}{l}\text { Analgésicos opioides equivalentes } \\
\text { de morfina }\end{array}$ & 30 minutos & Analgésicos e Reiki \\
\hline
\end{tabular}

Fonte: Autores (2021).

No Quadro 3 são associados os resultados das investigações às conclusões consolidadas.Pela relação entre os resultados dos estudos e as conclusões dos autores, notou-se que os analgésicos tiveram semelhança em termos de eficácia na recuperação do estresse pós-traumático. Além disso, percebeu-se que a associação entre esses e a terapia de Reiki potencializa a eficácia da diminuição da dor, estresse, níveis de ansiedade e tempo de medicação. Em alguns estudos, também se deparou com melhora dos sinais vitais.

Quadro 3: Resultados dos estudos selecionados e suas posteriores conclusões.

\begin{tabular}{|c|c|c|}
\hline Autores/ano & Resultados & Conclusão \\
\hline $\begin{array}{l}\text { Baldwin } \quad \text { et } \\
\text { al. }(2017)\end{array}$ & $\begin{array}{l}\text { O grupo de Reiki necessitou de menor número de } \\
\text { doses de medicação para controle da dor quando } \\
\text { comparado com pacientes submetidos ao Sham Reiki } \\
\text { e terapia convencional }\end{array}$ & $\begin{array}{l}\text { Estudo evidenciou pela primeira vez que o Reiki diminui } \\
\text { a dor, o estresse e os níveis de ansiedade em pacientes } \\
\text { hospitalizados submetidos à cirurgia de cirurgia de } \\
\text { substituição total do joelho. }\end{array}$ \\
\hline $\begin{array}{l}\text { Bourque et al. } \\
(2012)\end{array}$ & $\begin{array}{l}\text { O estudo revelou que } 16 \% \text { dos que receberam Reiki, } \\
\text { junto com a administração intravenosa de sedação } \\
\text { consciente, receberam menos de } 50 \mathrm{mg} \text { de meperidina } \\
\text {. }\end{array}$ & $\begin{array}{l}\text { Os resultados deste estudo piloto sugerem que pode haver } \\
\text { uma diminuição da meperidina necessária durante a } \\
\text { colonoscopia de triagem quando os pacientes recebem } \\
\text { Reikitratamentos antes do procedimento. Um estudo } \\
\text { maior desenvolvido para detectar diferenças menores de } \\
\text { medicação é o próximo passo para determinar com mais } \\
\text { precisão o efeito do Reiki no controle da dor. }\end{array}$ \\
\hline $\begin{array}{l}\text { Midili } \\
(2016)\end{array} \quad$ al. & $\begin{array}{l}\text { Observou-se que o grupo de Reiki usou menos } \\
\text { analgésicos durante o estudo e precisou deles depois } \\
\text { de um tempo mais longo doque os grupos de Reiki } \\
\text { falso e de controle }(\mathrm{p}<.05) \text {. }\end{array}$ & $\begin{array}{l}\text { O Reiki aplicado por } 15 \text { minutos na área da incisão após } \\
\text { umacesariana teve os efeitos esperadosna dor e na } \\
\text { necessidade do uso de analgésicos, mas não teve nenhum } \\
\text { efeito sobre os sinais vitais. }\end{array}$ \\
\hline $\begin{array}{l}\text { Midilli e Eser } \\
(2015)\end{array}$ & $\begin{array}{l}\text { Foi observado que os pacientes que utilizaram a } \\
\text { terapia de Reiki houve uma diminuição da } \\
\text { necessidade de analgésicos. }\end{array}$ & $\begin{array}{l}\text { O estudo mostrou a eficácia do Reiki na diminuição da } \\
\text { dor, frequência respiratória e ansiedade, como também na } \\
\text { redução da necessidade de analgésicos. }\end{array}$ \\
\hline $\begin{array}{l}\text { Notte } \\
(2016)\end{array} \quad$ et $\quad$ al. & $\begin{array}{l}\text { As sessões de terapia de Reiki resultaram em } \\
\text { reduções estatisticamentesignificativas da dor, exceto } \\
\text { aquelas sessões na Sala de Recuperação Pós- } \\
\text { Anestésica (SRPA). }\end{array}$ & $\begin{array}{l}\text { O Reiki pode ser um componente eficaz notratamento da } \\
\text { dor pós-operatória de pacientes cirúrgicos. }\end{array}$ \\
\hline
\end{tabular}


A qualidade das evidências, de acordo com o sistema GRADE está abordada no Quadro 4, em que três estudos obtiveram qualidade alta e dois estudos foram enquadrados em qualidade moderada.

Quadro 4: Avaliação da qualidade dos estudos selecionados para compor essa pesquisa- Fatores responsáveis pela diminuição da evidência.

\begin{tabular}{|l|c|c|c|c|c|}
\hline Autores & $\begin{array}{c}\text { Limitações } \\
\text { metodológicas }\end{array}$ & Inconsistência & $\begin{array}{c}\text { Evidência } \\
\text { indireta }\end{array}$ & Imprecisão & Qualidade \\
\hline Baldwin et al.(2017) & Presente & Ausente & Ausente & Ausente & Moderada \\
\hline Bourque et al. (2012) & Ausente & Ausente & Ausente & Ausente & Alta \\
\hline Midili et al. (2016) & Ausente & Ausente & Ausente & Ausente & Moderada \\
\hline Midilli e Eser (2015) & Presente & Ausente & Ausente & Ausente & Alta \\
\hline Notte et al. (2016) & Ausente & Ausente & Presente & & A \\
\hline
\end{tabular}

Fonte: Autores (2021).

\section{Discussão}

Na estatística, ciência que usa a análise dos dados para testar as hipóteses estatísticas, verifica-se o estudo da força da evidência clínica e, assim, se existem combinações e semelhanças entre grupos ou a veracidade de fenômenos de interesse. O pesquisador deve formular suposições, observar os fenômenos biológicos que ocorrem na população e retirar dessa população uma amostra para testar suas hipóteses. Nesse sentido, a similitude de uma amostra com a população que a originou permite que os resultados da análise dos dados sejam mais fidedignos para a elucidação das hipóteses (Rodrigues, Lima \& Barbosa, 2017).

Dessa forma, os estudos de Midilli et al. (2016) utilizaram o parâmetro $p$. Segundo, Ferreira e Patino (2015), o valor-p é definido como a probabilidade de se observar um valor da estatística de teste maior ou igual ao encontrado. Tradicionalmente, o valor de corte para rejeitar a hipótese nula é de 0,05 , o que significa que, quando não há nenhuma diferença, um valor tão extremo para a estatística de teste é esperado em menos de $5 \%$ das vezes. Esse valor vai determinar a significância de intervenção, e quanto mais próxima do valor zero, maior será a evidência do achado contra a hipótese nula.

Os resultados como descrito no quadro três ratificam a premissa de que o uso combinado entre o tratamento medicamento e a terapia de Reiki é uma estratégia eficiente na recuperação de pacientes submetidos a um estresse póstraumático (Bourque, Sullivan e Winter, 2012; Midilli \& Eser; 2015; Midili et al.,2016; Notte et al.,2016; Baldwin et al.,2017). Isso se dá, principalmente, devido à melhora significativa da dor que parece estar associada a uma recuperação mais rápida e com baixas doses de medicações analgésicas (Midilli \& Eser; 2015).

Diante do estudo de Midili et al.(2016), constatou-se que na aplicação do Reiki, o paciente é capaz de aliviar déficits ou bloqueios de energia no corpo, e assim, restaurando o equilíbrio energético e homeostático do organismo. Por conseguinte, entende-se que tal processo facilita a circulação do sangue e da linfa e estimula o sistema nervoso autônomo tocando os centros de energia do corpo, conhecidos por "chakra" com as mãos, ou em uma distância por meio de símbolos.

Nesse contexto, sabe-se que a terapia medicamentosa geralmente é tratada com opioides, os quais geralmente provocam reações adversas escalonadas de leve, moderada e alta. Complicações como hipoxemia, letargia e imobilidade podem inibir a capacidade do doente em deambular e curar (Notte et al., 2016) Tendo em vista a redução de tais situações, Midili et al. (2016) reafirmaram que a terapia do Reiki mostra-se como uma prática não invasiva e sem efeitos colaterais 
conhecidos, ausência de impactos negativos no tratamento e uma alternativa de baixo custo. No que tange ao às medições da frequência média de pulso e pressão arterial, as medidas dessas não foram significativamente diferentes umas das outras de acordo comos grupos e tempos $(\mathrm{P}>0.5)$

Pela análise descrita nos tipos de tratamento no quadro 2, na amostra estudada por Midili et al.(2016), notou-se que os resultados obtidos foram de significância muito alta $(\mathrm{p}<0,05)$ quando associado à terapia não medicamentosa. Os estudos afirmaram que noventa pacientes igualados por idade e número de nascimentos foram atribuídos aleatoriamente a um grupo de Reiki e/ou a um grupo controle (um descanso sem tratamento). O tratamento foi aplicado a ambos os grupos nas primeiras $24 \mathrm{e}$ 48 horas após o parto em um total de 10 a 30 minutos distribuído em regiões do corpo por 3 minutos cada.

Nesse ínterim, o Reiki foi aplicado por dois dias, sendo uma vez por dia (nas primeiras 24 e 48 horas) dentro de 4-8 horas após a administração de analgésico padrão, que foi administrado por via intravenosa por uma enfermeira. Uma escala visual analógica e o Inventário de Ansiedade do Estado foram usados para medir a dor e a ansiedade. Parâmetros hemodinâmicos, incluindo pressão arterial (sistólica e diastólica), pulso e respiração, as taxas e as necessidades de analgésicos também foram registradas. Em suma, os resultados mostraram que a aplicação de Reiki reduziu a intensidade da dor, o valor de ansiedade e a taxa de respiração, bem como a necessidade e o número de analgésicos. No entanto, não afetou a pressão arterial ou a pulsação. Reiki aplicação como uma intervenção de enfermagem é recomendada como uma dor e método de alívio da ansiedade em mulheres após parto cesáreo (Midili et al.,2016).

Sabe-se que a dor possui diferentes origens e, dentre elas, quando vivenciada no estresse pós-traumático vem acompanhada de outros sintomas, como ansiedade, aflição emocional, aumento da reatividade a estímulos estressantes, pensamentos negativos e importunos sobre o trauma que se não tratados adequadamente e manejados de uma forma que cure o paciente e não a doença pode levar a sérias complicações no futuro da pessoa. (Ferreira et al.,2018).

Dessa maneira, a dor em indivíduos com transtorno de estresse pós-traumáticopode causar desconforto, aumentar o estresse, apatia e ser gatilho para relembrar o trauma. Todavia, Ferreira et al.(2018) relataramque o trauma promove comportamento de hipervigilância e evita a prática de atividades relacionadas à dor que resultam na amplificação da experiência dolorosa.

No tratamento da dor utiliza-se o protocolo da Organização Mundial da Saúde (OMS), como afirmam Barbosa e Galriça Neto (2016). Os autores descrevem que a recomendação consiste em uma escala analgésica para o manejo da dor baseado na intensidade. Nessa conjuntura, um medicamento opioide muito utilizado no pós-operatório é a meperidina. Esta se liga a receptores opioides no sistema nervoso central inibindo a transmissão do impulso doloroso, como também apresenta atividade serotoninérgica. No manejo do pós-operatório na colonoscopia com o uso de tal medicação, segundo Bourque, Sullivan e Winter (2012) os resultados deste estudo sugeriram que pode haver a diminuição do uso da meperidina durante a colonoscopia de triagem em pacientes que recebem tratamentos de Reiki. Contudo, como é uma descoberta recente, um maior estudo deve ser desenvolvido para detectar diferenças menores de medicação entre os grupos e é a próxima etapa com mais precisão para determinar o efeito do Reiki no controle da dor.

Ademais, Baldwin et al.(2017) afirmaram que o resultado mais impressionante do estudo foi que o Reiki reduziu os escores de dor em 18 conteúdos submetidos à cirurgia de substituição do joelho único. Um procedimento que é bastante angustiante, doloroso e que demanda muito tempo no pós-operatório para o alívio da dor, o qual envolve protocolos de gerenciamento de dor fortes e invasivos.

Os autores citados ainda destacaram que a redução da dor em pacientes no ambiente hospitalar é uma importante dimensão de um ambiente de cuidado e cura e pode melhorar a experiência e a satisfação do paciente com cuidados e gestão da dor, influenciando os dados sendo coletados por hospitais para qualidade e regulamentação conforme estudado. 
Percebe-se, também que a redução da dor pós-operatória é um gatilho de oportunidades entre hospitais com base nos resultados do paciente, satisfação do paciente, e taxas de readmissão, sendo o Reiki, uma oferta de baixo custo com uma abordagem de cura e cuidado para o atendimento ao paciente. Assim, tem-se um impacto financeiro significativo para as seguradoras, pacientes e provedores. Além de que, o posicionamento do Reiki como um adjunto ao protocolo farmacológico da dor deve promover uma adoção e aceitação generalizada. Reiki é aditivo e pode aumentar a adesão do paciente, permitindo alta pontual e menos complicações.

De acordo com a avaliação das evidências feitas pelo sistema GRADE, pode-se concluir que há forte recomendação em relação aos dados que foram analisados e discutidos. Todavia, foi visto que houveram limitações relacionadas ao tamanho da população da amostra nas evidências, referidas pelos próprios pesquisadores, além de ser uma terapia a ser mais promissora para o futuro, visto que poucos conhecem sobre tal abordagem. Mas, ainda assim, pela análise estatística, a maioria dos resultados foi significativa, tornando-se como forte recomendação da intervenção como forma de tratamento alternativo.

\section{Conclusão}

No presente estudo concluiu-se que a terapia Reiki associada à terapia medicamentosa, como analgésicos e opioides, tem boa eficácia em pacientes com dor, ficando evidente sua vantagem no uso em indivíduos que precisam de analgesia. No entanto, faz-se necessário maior disseminação do conhecimento sobre tal terapia; bem como, pesquisas com amostras maiores. Apesar dos bons resultados, é sugerida pelos autores deste estudo, a realização de mais ensaios clínicos, para consolidar a total eficácia da associação com a terapia Reiki no tratamento da dor.

Sugerimos para pesquisas futuras que a terapia Reiki seja também estudada em associação com transtornos psicológicos, como ansiedade e depressão, em conjunto com a terapia medicamentosa. No intuito de avaliar se, nesses casos, também há melhoria na sintomatologia dos indivíduos afetados.

\section{Referências}

Akobeng, A. K. (2005). Principles of evidence based medicine. Arch. Dis. Child, 90 (8), 837-840.

Baldwin, A. L., Vitale, A., Brownell, E., Kryak, E., \& Rand, W (2017). Effects of Reiki on Pain, Anxiety, and Blood Pressure in Patients Undergoing Knee Replacement: A Pilot Study. HolisticNursingPractice, 31 (2), 80-89.

Barbosa, A., \& Galçira Neto, I (2016). Manual de Cuidados Paliativos. Núcleo de Cuidados Paliativos/Centro de Bioética da Faculdade de Medicina de Lisboa.

Bourque, A. L., Sullivan, M. E., \& Winter, M. R. (2012). Reiki as a pain management adjunct in screening colonoscopy. Gastroenterology nursing: the oficial jornal of the Society of Gastroenterology Nurses and Associates, 35 (5), 308-312.

Brasil. Ministério da Saúde. Secretaria de Ciência, Tecnologia e Insumos Estratégicos. Departamento de Ciência e Tecnologia. (2014). Diretrizes metodológicas: Sistema GRADE-Manual de graduação da qualidade da evidência e força de recomendação para tomada de decisão em saúde. Ministério da Saúde.

De-La-Torre-Urgate-Guanilo, M. C., Takahasi, R. F., \& Bertolozzi, M. R (2011). Revisão sistemática: noções gerais. Revista da Escola de Enfermagem da USP, 45 (5), 1260-1266.

Ferreira, D. M. A. O., Vaz, C. C. O., Stuginski-Barbosa, J., \& Conti. P. C. R. (2018). Post-traumatic stress disorder and temporomandibular dysfunction: a review and clinical implications. BrazilianJournalofPain, 1 (1), 55-59.

Ferreira, J. C., \& Patino, C. M (2015). What does the value really mean? Jornal Brasileiro de Pneumologia, 41 (5),485-485.

Freitag, V. L., Andrade, A., \& Badke M. R. (2015). O Reiki como forma terapêutica no cuidado à saúde: uma revisão narrativa da literatura. Revista eletrônica trimestral de enfermagem, p.346, 1695-6141.

Freitag, V. L., Dalmolin, I. S., Badke, M. R., \& Andrade, A. (2014).Benefitsof Reiki in older individual swith chronic pain.Texto e context enfermagem, , 23(4): 1032-40.

Galvão, M. C. B., \& Ricarte, I. L. M. (2019). Revisão sistemática da literatura: conceituação, produção e publicação. LOGEION: Filosofia da informação, 6 (1), 57-73. 
Research, Society and Development, v. 10, n. 9, e7510917807, 2021

(CC BY 4.0) | ISSN 2525-3409 | DOI: http://dx.doi.org/10.33448/rsd-v10i9.17807

Galvão, T. F., Pansani, T. S A., \& Harrad, D. (2015). Principais itens para relatar revisões sistemáticas e meta-análises: A recomendação PRISMA. Epidemiologia e Serviços de Saúde, 24 (2), 335- 342.

Midilli, T. S., Ciray, N., Gunduzuglu, N. C. (2016). Effects of Reiki on pain and vital signs when applied to the incisión área of the body after cesarean section surgery. HolisticNursingPractice, 30 (6), 368-378.

Midilli, T. S., \& Eser, I (2015). Effectsof Reiki on Post-cesarean Delivery Pain, Anxiety, and Hemodynamic Parameters: A Randomized, Controlled Clinical Trial. PainManagNurs, 16 (3), 388-399.

Notte, B. B., Fazzini, C., \& Mooney, R. A (2016). Reiki's effect on patients with total knee arthroplasty: a pilot study. Nursing,46 (2), 17-23.

Rodrigues, C. F. S., Lima, F. J. C., \& Barbosa, F. T. (2017). Importância do uso adequado da estatística básica nas pesquisas clínicas. BrazilianJournalOfAnesthesiology, 67 (6), 619-625.

Santos, C. B. R., Gomes, E. T., Bezerra, S. M. M. S., \& Püschel, V. A. A. (2019). Reiki protocol for preoperative anxiety, depression, andwell-being: a nonrandomized controlled trial. RevEscEnferm USP. 2020,54:e03630

Spezzia, S., \& Spezzia, S. (2018). O uso do reiki na assistência à saúde e no sistema único de saúde. Revista De Saúde Pública Do Paraná, 1(1), 108-115.

Thrane, S., \& Cohen, S. M. (2014). Effect of Reiki therapy on pain and anxiety in adults: anin-depth literatura review of randomized trials with effect size calculations. Pain Management Nursing, 15 (4), 897-908.

Weze, C., Leathard, H., \& Stevens, G (2005). Evaluation of healing by gentle touch. PublicHealth, 119 (1), 3-10. 